\section{MEINUNG}

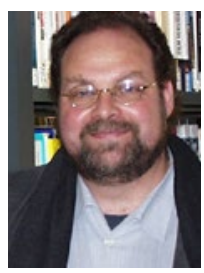

Die richtigen

Schlüsse aus den politisch gewollten Herausforderungen zu ziehen ist eine Kunst, die auch Mut erfordert. Seit Jahrzehnten wird über das Problem des TheoriePraxis-Transfers diskutiert. Das Gesundheitszentrum Glantal begegnet diesen Herausforderungen mit dem Projekt „Implementierung von Pflegeexperten." Die wissenschaftliche Begleitung findet durch die Ernst-Abbe-Hochschule Jena statt.

Um eine effiziente und qualitativ hochwertige Pflege auch in $\mathrm{Zu}$ kunft, bei steigenden Anforderungen und Anzahl multimorbider Patienten sicherzustellen, ist eine evidenzbasierte Pflegepraxis Grundvoraussetzung. Die schulisch ausgebildeten Pflegekräfte müssen gezielt unterstützt werden. Dieser Ansatz ist nicht neu, bedauerlicherweise geht die Umsetzung im deutschen Gesundheitswesen sehr langsam voran. Seit Jahren wird auf Hochschulebene Pflegepersonal für die erweiterte Pflegepraxis ausgebildet - nur in seltenen Fällen finden diese im Anschluss entsprechende Einsatzfelder.

Im Gesundheitszentrum Glantal wurde deshalb ein Modell entwickelt, dass genau diese Zielgruppe im Fokus hat und durch die verschiedenen Kompetenzen der Bachelor- und Masterabsolventen eine evidenzbasierte Pflege in der Pflegepraxis gewährleisten soll. Durch den flächendeckenden Einsatz von akademisch ausgebildetem Pflegepersonal in der direkten Patientenversorgung wird somit eine wesentliche Lücke im TheoriePraxis-Transfer geschlossen. Die Kollegen im Gesundheitszentrum Glantal leisten mit dem Projekt "Implementierung von Pflegeexperten" Pionierarbeit für die deutsche Pflegeprofession.

Prof. Dr. Olaf Scupin

Ernst-Abbe-Hochschule Jena

\title{
Evidenzbasierte Pflegepraxis
}

\section{Pflegeexperten als Wegbereiter}

\author{
Unter dem demografischen Wandel und der Zunahme von Multimorbi- \\ dität wächst der quantitative und qualitative pflegerische Aufwand ste- \\ tig. Das Gesundheitszentrum Glantal in Rheinland-Pfalz möchte diesen \\ Herausforderungen mit der Implementierung hochschulisch ausgebilde- \\ ter Pflegekräfte in der direkten Patientenversorgung entgegentreten.
}

$\mathrm{D}$ ie Anforderungen im Gesundheitswesen steigen kontinuierlich. Neben dem Verhältnis von Pflegebedürftigen zu den Pflegenden nimmt auch die Anzahl an hochbetagten und somit mehrfach oder chronisch erkrankten Menschen unter den Pflegebedürftigen zu. Nach einer Berechnung des Statistischen Bundesamtes ist das Alter mit der Fallzahl an Krankenhausbehandlungen eng verbunden. $49 \%$ aller Krankenhausbehandlungen betreffen aktuell die Altersklasse ab 60 Jahre, Tendenz steigend. Durch die immer komplexeren Fälle sind die Pflegenden nicht nur mit dem für den Krankenhausaufenthalt ursächlichen Problem konfrontiert. Der Patient bringt auch weitere Diagnosen und Pflegephänomene mit. Diese müssen beherrscht und mit Blick auf die aktuellsten pflegewissenschaftlichen Erkenntnisse behandelt werden.

Zusätzlich verschärfen sich die Rahmenbedingungen für die Pflege dramatisch und der ökonomische Druck steigt. Pflegefachkräfte sehen in ihrer täglichen Arbeit den Bedarf an evidenzbasierter Pflegepraxis und wünschen sich eine fachliche Unterstützung. Aus Hochschulsicht wurde auf diese Voraussetzungen reagiert. Während zu Beginn der Akademisierung der Schwerpunkt auf der Qualifizierung von Lehr- und Leitungspersonen lag, steht aktuell die erweiterte Pflegepraxis im Fokus. Ziel dieser Entwicklung ist eine gesteigerte Versorgungsqualität und ein verbessertes Patientenoutcome.

\section{Akademisch ausgebildete Pflege wird zu wenig genutzt}

Trotz des geänderten Schwerpunkts in der akademischen Ausbildung und der Anforderungen aus der Praxis finden sich nur vereinzelt Absolventen von Studiengängen, die bewusst in der direkten Pflege eingesetzt werden. In Meisenheim möchten wir neue Wege beschreiten und diese Lücke zwischen Theorie und Praxis durch den gezielten Einsatz akademisch ausgebildeter Pflegenden schließen. Ingolf Drube, Pflegedirektor des Gesundheitszentrums Glantal, ist überzeugt, dass das Potenzial der akademisch ausgebildeten Pflegekräfte im direkten Patientenkontakt zur Umsetzung des notwendigen Theorie-Praxistransfers genutzt werden muss. Ein entsprechendes Konzept wird derzeit wissenschaftlich begleitet implementiert. Ziel ist es, zukünftig eine erweiterte Pflegepraxis abzubilden. Innovative Versorgungsformen im Bereich von Delegation und Substitution können so beispielhaft umgesetzt werden.

\section{Die Ausgangslage in Meisenheim}

Das Gesundheitszentrum Glantal ist ein Zentrum für Akutversorgung in den Fachbereichen Neurologie, Chirurgie und Innere Medizin, Neurologische (Früh-)Rehabilitation und zur Therapie von Kommunikationsstörungen bei Kindern und Jugendlichen mit insgesamt 207 Betten und Plätzen in Trägerschaft des Landeskrankenhauses (AöR). Die Krankenhausleistungen werden im 